\title{
Design Space Exploration of Drone Infrastructure for Large-Scale Delivery Services
}

\author{
Sangyoung Park, Licong Zhang, Samarjit Chakraborty \\ Institute for Real-Time Computer Systems, Technical University of Munich \\ \{sangyoung.park,licong.zhang@tum.de,samarjit\}@tum.de
}

\begin{abstract}
Drones, also referred to as unmanned aerial vehicles (UAVs), are recently expanding their field of usage beyond military surveillance and tactical applications. Commercial drone delivery service is one of the promising applications in the near future, and a number of companies are already pushing forward the legal and technical barriers to realize the concept. Unlike conventional applications of drones, the success of a commercial application depends critically on the profitability. The major sources of expense are the electricity cost and battery purchasing cost due to aging. Hence, it is crucial to maximize the energy efficiency and mitigate battery aging of the drone delivery business. However, no prior work has extensively assessed the problem for the business as a whole. This paper, for the first time, proposes a holistic and detailed analysis on the profitability and time to delivery of the drone delivery business. This paper identifies the major design parameters and runtime management potentials that affect the profitability and time to delivery of the business. We have implemented a discrete event simulator based on detailed models of the comprising components. We perform a design space exploration to understand the effects of the various battery configurations, battery attachment technique, drone flight speed, etc., on time to delivery, electricity cost, and battery purchasing cost. Our results show that such control knobs have a significant impact on the time to delivery and the operating cost of the business.
\end{abstract}

\section{Categories and Subject Descriptors}

C.3 [Special-purpose and application-based systems]: Real-time and embedded systems

\section{INTRODUCTION}

Use of drones, or unmanned aerial vehicles (UAV), has been restricted mostly to military surveillance and tactical applications over the last decade. A number of enterprises such as Amazon are

\footnotetext{
*This work is supported by the Bavarian Ministry of Economic Affairs and Media, Energy and Technology as part of the EEBatt project.
}

Permission to make digital or hard copies of all or part of this work for personal or classroom use is granted without fee provided that copies are not made or distributed for profit or commercial advantage and that copies bear this notice and the full citation on the first page. Copyrights for components of this work owned by others than ACM must be honored. Abstracting with credit is permitted. To copy otherwise, or republish, to post on servers or to redistribute to lists, requires prior specific permission and/or a fee. Request permissions from permissions@acm.org.

ICCAD '16, November 07-10, 2016, Austin, TX, USA

(c) 2016 ACM. ISBN 978-1-4503-4466-1/16/11 . \$ $\$ 15.00$

DOI: http://dx.doi.org/10.1145/2966986.2967022

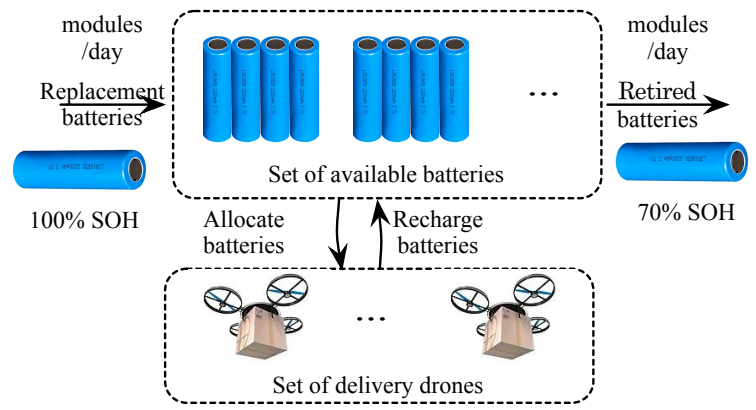

Figure 1: Battery flow of a drone delivery service.

looking into the potential of commercial delivery drones. Amazon Prime Air plans to use multirotor UAVs to autonomously deliver packages weighing below 5 pounds to its customers within 30 minutes of ordering [3]. Logistics company DHL has demonstrated a practical scenario of using delivery drones [2]. However, a number of technical challenges as well as legal ones prevent the realization of the business. The aspect of drone design, autonomous flight, and coordination of drones are assessed from prior works [12, 4, 7]. However, no work has focused on the profitability and time to delivery aspect from the perspective of the whole business. Many battery powered vehicles, e.g., electric vehicles and drones, suffer from disadvantages coming from high price of batteries, low energy density and limited lifetime. Batteries for drones suffer from even harsher environment compared with electric vehicles. Delivery drones are inherently more weight sensitive than electric vehicles, and this forces them to attach the minimum amount of batteries and exploit the full capacity. They are also designed to fly for less than 30-40 minutes leading to average discharge rates of up to 2-3C, which is much higher than electric vehicles. Large depth-ofdischarge (DoD) and discharge rate of batteries leads to fast aging and battery replacement costs will be one of the major factors that undermines the profitability of the service.

Battery management of a drone delivery service for our problem setup is summarized in Figure 1. Delivery service provider maintains a certain number of delivery drones and a set of batteries to power the drones. Batteries are automatically detachable from the drones for re-charging and drones can make the next flight immediately with an already charged battery modules similar to the recently proposed prototype [16]. This can increase the utilization of drones drastically compared with batteries fixed to drones. According to the differing requirements of each delivery task, e.g., payload weight, distance to the destination, batteries are allocated to each drone and serve the tasks. After their service, allocated batteries are recharged for the next service. As the time goes by, the stateof-health $(\mathrm{SOH})$ of batteries degrades and they are replaced with an equal number of fresh batteries when the end of life is reached. Bat- 
tery retirement and replacement will be a regular event, and battery replacement costs will constitute a major portion of the operating costs as well as the electricity costs.

Although there has been some research on design and navigation of the drones, not many works have viewed the feasibility of the drone delivery service from the perspective of business as a whole. A number of open design optimization problems remain in this domain such as the number and types of batteries that should be maintained, the number of drones, battery attachment strategy, battery charging strategy, according to varying delivery request patterns and payload distributions. In this paper, we design a discrete event simulator focused on the power and energy consumption perspective based on elaborate models of battery and drone power models to explore design parameters mentioned above to analyze their impact on the electricity cost, battery purchasing costs, and time to delivery.

\section{RELATED WORKS}

There have been several related works addressing the problem of using drones for delivery service. Logistics company DHL has demonstrated a practical scenario of the delivery drones and highlighted the technical hurdles [2]. [12] has developed an autonomous framework for delivery drones and $[4,7]$ proposed some navigation and control technology. [16] considered the case where batteries are automatically detachable from the drones for re-charging and the drones can make the next flight immediately with already charged battery modules, which is similar to the battery model and charging strategy that we use in this paper. However, the aforementioned related works focused more on the technical and legal feasibility of the drone delivery service and not on the economical feasibility, i.e., the profitability, which is the focus of this paper at hand.

Towards the profitability issue, the cost is considered by [6] as one of the most important challenges for the feasibility of the drone delivery service, amongst other ones including vehicle design, navigation and coordination. It identified the electricity cost and battery cost as the most important factors in terms of the cost of the delivery service and has conducted a cost estimation. But this estimation is based on high-level models and does not consider the impact of various factors like battery aging, heterogeneous batteries, etc. A profitability analysis has been done for a similar application, a fleet of electric vehicles [9]. However, the nature of the problem itself is different. That work assumes that the fleet owner benefits from fluctuating grid electricity price whereas the current paper benefits from delivery services. Another work performs a very similar economic analysis on a taxi fleet with battery swapping stations and uses genetic algorithm to maximize the profit of the fleet operating company [14]. However, this work is based on very simple cycle model such that it cannot test detailed management policies. In comparison, in our work, we can test and evaluate different management policies using the proposed simulator.

\section{DESIGN AND MANAGEMENT OF DRONE DELIVERY SERVICES}

In this section, we describe the major components of a drone delivery platform and enumerate design criteria. The major components comprising the business are shown in Figure 2. Customers order products online and make delivery requests. Then, the ordered product is taken from the product storage and packaged for drone delivery. A charged battery is attached to the drone at a battery replacement platform. Drone delivers the packet to the customer and returns to the drone delivery platform. The battery is

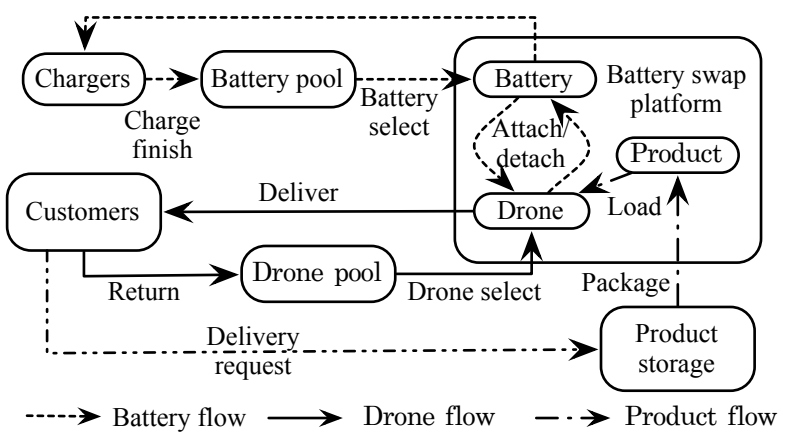

Figure 2: Major components and battery, product flow of a drone delivery business.

detached from the drone and re-charged for the next mission, while the drone leaves immediately for the next delivery with a swapped battery.

Objective: There could be multiple objectives of the drone delivery business. Probably the most important objective would be to maximize the profit of the business under a given time to delivery constraint. Also, it would be possible to minimize the time to delivery under a fixed budget.

There are multiple design parameters that affect the objectives. Number of batteries/drones/chargers: The most important design time parameters include the total number of batteries, the number of drones, the number of chargers to serve delivery requests. If the number of battery sets is too large, the initial investment cost will be high, and battery depreciation due to calendar aging will increase the operating cost of the business. On the other hand, if the number is too small, packets and drones might have to wait for a battery to finish charging, increasing the time to delivery. Similar principle holds for the number of drones and chargers.

Heterogeneous batteries/drones: Also, heterogeneous types of batteries could be used. As it is quite likely for the distance to destination and payload weight differ from delivery request to request. However, attaching an unnecessarily large battery to a drone serving a delivery request requiring only small energy could be an overkill due to its excessive weight. Thus, it might be a good idea to use heterogeneous types of batteries to serve delivery request with differing energy requirements. Another thing to note is that the number of customer orders will not be constant, but likely to change over time. While the peak and valley of the volume of customer orders could differ drastically, the delivery business should be able to maintain constant time to delivery. However, if the discussed design parameters are determined to satisfy the time to delivery constraint for the worst-case scenarios, this might be an overkill initial investment that severely harms the profitability.

There are also runtime management techniques available.

Battery charging: The decision of when and how to charge the batteries could affect the objectives. For example, it is generally perceived that higher average state-of-charge (SOC) is harmful for the battery life. If it is known when a battery will be discharged, in our case when the battery will be used for a delivery task, charging could be delayed as much as possible. A similar idea to extend the battery life has been proposed for smartphones [15]. Charging speed could also be a control knob for optimization. If a battery is charged at higher rate, it would be able to serve more number of flights per day. On the other hand, energy efficiency of charging would be lower due to the rate capacity effect of batteries.

Battery attachment: We define the term battery attachment as a mapping from a set of batteries to a set of drones, which corresponds to attaching a battery to a drone. In the case of using heterogeneous types of batteries, the decision of which batteries to at- 
tach to drones affects the objectives. Carrying just the right amount of battery would be ideal, but due to practical reasons, the types of available batteries will be limited, which forbids the drones to carry the exact amount.

Drone selection: We might even have a heterogeneous types of drones. Heavier and high-power drones for weighty packets, and lighter low-power drones for lightweight packets.

Battery replacement: Battery replacement strategy involves the decision of when to retire a battery from the battery pool and replace it with a newly purchased battery. There will be a trade-off relationship between the purchasing cost and time to delivery as will be described in the subsequent sections.

\section{IMPACT OF BATTERY AGING}

Battery capacity fading is one of the major consequences of $\mathrm{SOH}$ degradation. Battery powered vehicles such as electric vehicles or drones are very weight sensitive, so that capacity fading directly impacts the performance of the vehicles. Capacity fading is caused both by calendar aging and cycle aging. Calendar aging depends on the ambient temperature and the average SOC of the battery. Cycle aging occurs when the battery is in use. The aging rate depends significantly on the SOC deviation and the charging/discharging rate. As we are tackling the $\mathrm{SOH}$ degradation of the batteries for drones, it is important that we use an accurate and elaborate SOH degradation model to verify our proposed idea. An elaborate model based on theory of crack propagation in structural materials applied to the battery electrodes, considers all these effects and calculates capacity fading over time [13]. The model calculates capacity fading cycle-wise. First, the model derives the average SOC, $\overline{S O C}$, over a cycle as follows.

$$
\overline{S O C}=\left(\int_{T_{m}} \operatorname{SOC}(t) d t\right) / T_{m},
$$

where $T_{m}$ is the duration of a cycle. The normalized SOC deviation is calculated by

$$
\sigma=2 \sqrt{3 \int_{T_{m}}(S O C(t)-\overline{S O C})^{2} d t / \int_{T_{m}} d t}
$$

Factor $2 \sqrt{3}$ is to normalize the standard deviation to 1 for a full cycle. The effective number of throughput cycles is denoted by $N$ :

$$
N=\int_{T_{m}} \frac{|i(t)| d t}{2 Q_{n o m}}
$$

We calculate the intermediate parameters $L_{1}$ and $L_{2}$ for

$$
\begin{gathered}
L_{1}=K_{c o} N \cdot \exp \left((\sigma-1) \frac{T_{r e f}+273}{K_{e x}\left(T_{B}+273\right)}\right)+0.2 \frac{t_{\text {cycle }}}{t_{\text {life }}}, \\
L_{2}=L_{1} \exp \left(4 K_{\text {soc }}(\overline{S O C}-0.5)\right)(1-L),
\end{gathered}
$$

where $K_{c o}$ is a normalization coefficient for $N$ and $K_{e x}$ is a constant exponent for depth of discharge. $T_{r e f}$ is the reference battery temperature of $25^{\circ} \mathrm{C}$ and $T_{B}$ the battery temperature. $t_{\text {cycle }}$ is the duration of one cycle and $t_{\text {life }}$ the shelf life at $25^{\circ} \mathrm{C}$ and $50 \%$ SOC until $80 \%$ of the initial capacity remain. Parameter $L_{2}$ reflects the effect of average SOC with coefficient $K_{s o c}$. The above mentioned parameters were derived by [13] for the A123 ANR26650M1A cell. Finally, the total increase in the life parameter is given by

$$
L\left(T_{m}\right)=L_{2} \exp \left(K_{t}\left(T_{B}-T_{r e f}\right) \frac{T_{r e f}+273}{T_{B}+273}\right),
$$

where $K_{t}$ accounts for a doubling of the decay rate for each $10^{\circ} \mathrm{C}$ rise in temperature. Summing up the damage done by each cycle, we calculate the remaining life of the battery.

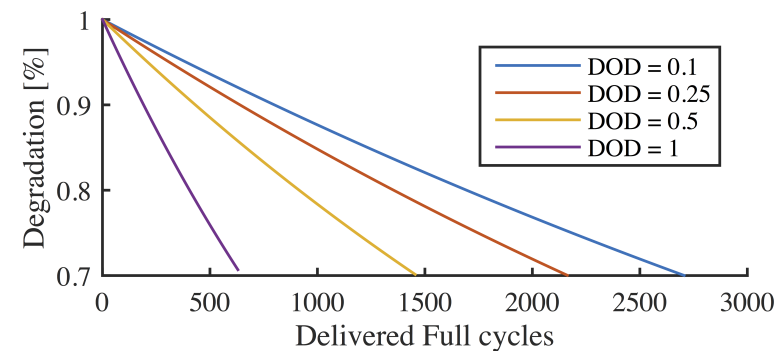

Figure 3: Battery capacity fading according to different DoD (fully charged each cycle) using the model from [13].

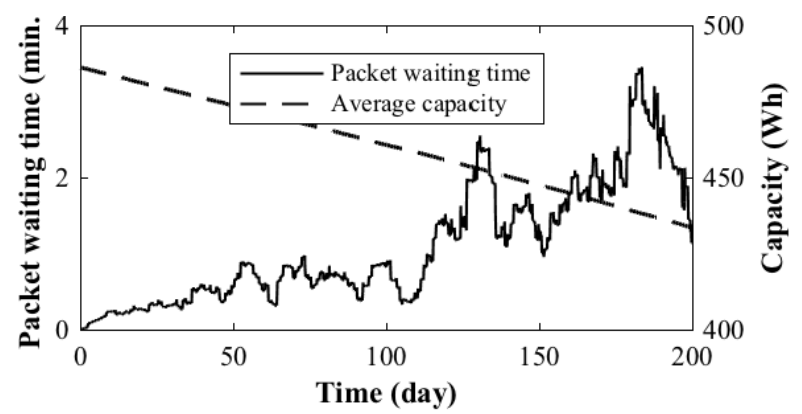

Figure 4: The impact of battery aging on time to delivery.

Battery aging has effects on various aspect of the drone delivery business. Degraded cycle efficiency due to increased internal resistance leads to increased cost in electricity bill. Degraded capacity of a battery has profound impact on the packet waiting time defined as the time a packet is waiting for a drone or a battery pack to be ready for service. First, the number of batteries that are capable of serving long-distance delivery decreases, and hence, such delivery requests have to wait for a longer time. Second, degraded capacity forces the battery to discharge more deeply, which increases the average charging time. We provide an averaged packet waiting time in Figure 4.

\section{COMPONENT MODELS}

In this section, we provide the component models used for the discrete-event simulator to evaluate the various costs related to the drone delivery business.

Battery equivalent circuit model: We use a widely-known battery equivalent circuit model shown in Figure 5, where the parameters in the figure is given as follows [5].

$$
\begin{aligned}
V_{O C}(S O C) & =\alpha_{11} e^{\alpha_{12} S O C}+\alpha_{13} S O C^{3} \\
& +\alpha_{14} S O C^{2}+\alpha_{15} S O C+\alpha_{16}, \\
R_{S}(S O C) & =\alpha_{21} e^{\alpha_{22} S O C}+\alpha_{23} \\
R_{t s}(S O C) & =\alpha_{31} e^{\alpha_{32} S O C}+\alpha_{33} \\
R_{t l}(S O C) & =\alpha_{41} e^{\alpha_{42} S O C}+\alpha_{43} \\
C_{t s}(S O C) & =\alpha_{51} e^{\alpha_{52} S O C}+\alpha_{53} \\
C_{t l}(S O C) & =\alpha_{61} e^{\alpha_{62} S O C}+\alpha_{63}
\end{aligned}
$$

Drone power consumption: Battery powered drones, in general, have limited flight time around 30 minutes and are very weight sensitive. The flight time depends significantly on the battery and payload weight. Flight time decreases as much as to $50 \%$ according to the payload [1]. Battery weight and payload weight should be chosen very carefully as it affects not only the energy efficiency, but also the flight time and flight range. Drone power consump- 


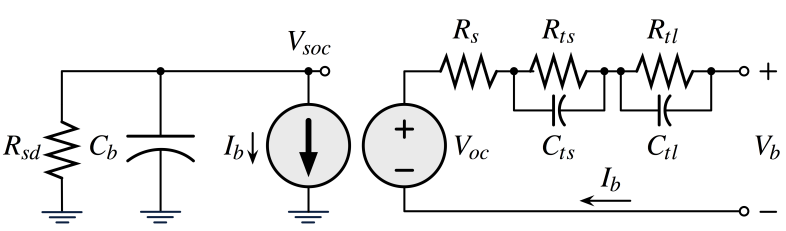

Figure 5: Equivalent circuit model of a battery cell [5].

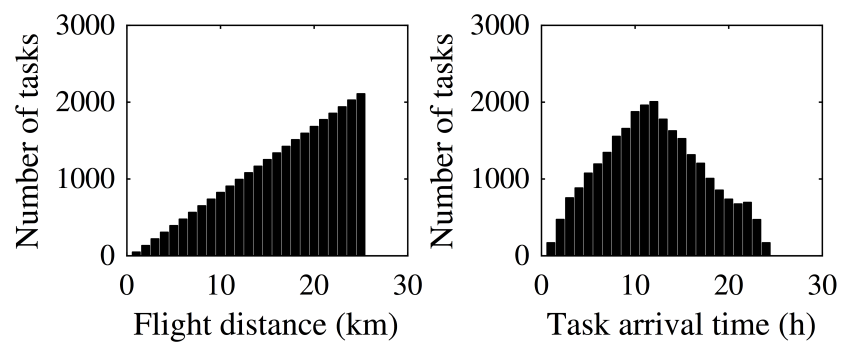

Figure 6: Delivery task distribution according to flight distance and arrival time per day where bin sizes are $1 \mathrm{~km}$ and $1 \mathrm{~h}$.

tion depends significantly on types of vehicles and operating conditions, and a number of models exist [1]. We use the following drone power model from [8] and use parameters from [11].

$$
\begin{aligned}
P_{\text {hover }} & =\frac{(2 M g)^{3 / 2}}{4 \sqrt{\rho A}}, \\
P_{\text {drag }} & =\frac{1}{2} \rho C_{d} v^{3} A_{f}, \\
A_{f} & =A_{h} \cdot \sin \theta+A_{v} \cdot \cos \theta, \\
\theta & =f(v),
\end{aligned}
$$

where $P_{\text {hover }}$ is the hover power, $M$ is the total weight of the drone including battery and payload, $g$ is the gravitational acceleration, $\rho$ is the air density, and $A$ is the total area of the rotors. Drone angle, $\theta$, is a function of the drone speed [17]. This is a very rough power consumption model of a drone and it might differ from practical cases where there is wind and other forces affecting the operation of the drone. However, we emphasize that our technique does not rely on the details of the power consumption model as long as the major trade-off relationships, such as payload vs. flight time, hold.

Charger: We should also accurately quantify the effect of DCDC converters in the modular battery system architecture. One of the major drawbacks preventing the use of the proposed architecture is its complexity and energy efficiency decrease by adding additional DC-DC converters in the power path. Well designed DCDC converters generally exhibit maximum conversion efficiency of $90 \%$ to $95 \%$, but its value changes according to input/output voltage and current. For our case, the input and output voltage is roughly fixed and does not change dynamically over a short period of time. Therefore, without loss of generality, we use a curvefitted converter efficiency model taking only the load current into account, where the efficiency value changes from $85 \%$ to $94.9 \%$, which corresponds well with the values of commercial products.

Task distribution: We assume that the distribution of the delivery request arrival time is not uniform over time of day, and it will have peaks and valleys. Figure 6 shows the distribution of the delivery task arrival time, which is used throughout the paper. Delivery requests are made starting at 9 AM until 6 PM. We state that our simulator and observed results do not dependent on particular distribution. The reason we have chosen linear distribution for flight distance is that it would be the case if the area around the packet station is uniformly populated and makes uniform number of request per area. However, in practical scenarios, this distribu-

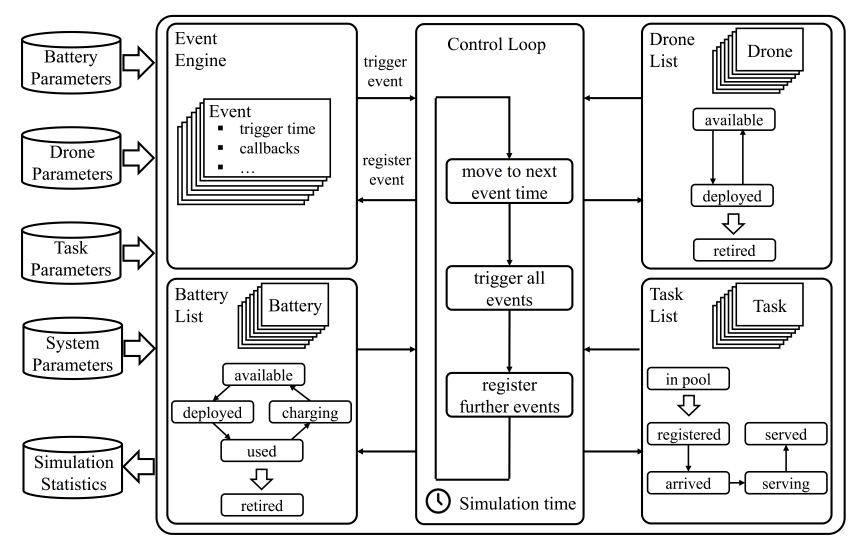

Figure 7: Architecture and control flow of the simulator.

tion might not be the case because the infrastructure would require a significant space for storing products, and hence, be located at outskirts of a city. The same goes for the task arrival time distribution. The distribution of delivery requests according to time of day might not necessarily follow the distribution shown in Figure 6, but we reiterate that our framework is not dependent on the particular distributions. The total number of delivery requests per day we have assumed is about 30,000, a value borrowed from data of a large home-shopping company located in Dedemsvaart occupying $33,000 \mathrm{~m}^{2}[10]$.

\section{DELIVERY DRONE BATTERY SIMULA- TOR}

Towards the evaluation of various metrics related to the drone delivery business, we have developed a discrete event simulator in the context of this paper. Although there exist different frameworks for discrete event simulation, in order to minimize the overhead, we have developed our own simulator in $\mathrm{C}++$ with support for mathematical calculations from the GNU Scientific Library (GSL). The architecture of the simulator is shown in Figure 7. The simulator consists mainly of four modules, namely the event engine, the battery list, the drone list and the task list. The battery list, drone list and task list manage a set of batteries, drones and tasks and their state transition respectively. The simulator is driven by the event engine, where events can be registered and triggered each at a specific time point in the simulation.

Batteries, drones and tasks: The battery list keeps track of a set of batteries, which can be in one of the following states: available, deployed, used, charging and retired. A battery in the available state is fully charged and ready to be attached to drones. The deployed state denotes that the battery is currently attached to a drone in delivery. Once the drone returns, the battery is transferred to the used state and waits to be recharged. If there is a charger available, the battery can then be recharged (charging state) and afterwards becomes available again. A battery maintains some internal variables like the SOC and parameters like the capacity. The time that a battery stays in the deployed state depends on the flight time of a specific task and can be calculated according to the characteristics of the tasks and the SOC of the battery. The charging time can be calculated by the current SOC, the battery capacity and the charging current and voltage. After each charging cycle, the battery capacity degradation and the aging mechanism are computed. If the $\mathrm{SOH}$ deteriorates below a certain threshold, the battery is no longer considered fit for usage and thus moved to the retired state. New batteries can also be added to the list before the simulation starts or during the simulation. The drones are managed by the 
drone list module and can switch between the available, deployed and retired state. The available and deployed state represent that the drone is available for a delivery task or is currently serving a task respectively. Similar to the batteries, if the performance of a drone deteriorates, it can also be moved to the retired state and new drones can be added to the simulation. A task instance is a four tuple of order time, energy required for delivery, payload weight and deadline for delivery. A task can firstly be registered (registered state) so that it is known to the simulator's event engine. It becomes arrived once the simulation time arrives at the pre-scheduled arrival time of the task. Once a task arrives, if there are resources (drones and batteries) available to make the flight, it will transit into the serving state. After the flight time is finished, the task is moved to the served state. Task series can be pre-generated before the simulation starts or generated during the simulation. In the former case, the task series can be loaded into the simulator at the beginning and put into the inpool state. The tasks can then be registered during the simulation based on different metrics (e.g., a fixed window of time ahead). This implementation prevents the event engine to be overloaded with tasks, in which case the simulation speed could be slower.

Simulator engine and control flow: The control flow of the simulator is based on the event engine, where a list of events, their triggering time and callback functions are maintained. The simulator executes an indefinite loop until the event list is empty. In each cycle, the simulation time proceeds to the next event triggering time, processes all events and then registers further events if there is any. Examples of events include: (i) task arrival - a task is scheduled to arrive, (ii) task service ends - the service of a delivery task is scheduled to end, and (iii) charging finishes - the charging of a battery is scheduled to end.

\section{DESIGN SPACE EXPLORATION OF DRONE INFRASTRUCTURE}

There are a number of design parameters which affect the profitability of the delivery business, for example, how many battery modules and drones we should maintain according to different volumes of delivery request. We analyze the relationship between the following parameters to identify the appropriate value. This problem is a multi-objective problem where the objectives are the average packet waiting time, battery purchasing cost, electricity usage, and so on.

\subsection{Simulator Validation}

Short-term simulation results: We first show the simulation results in general. Figure 8 shows the results for two days in the middle of a longer simulation. The simulation used 1,900 batteries of 452.36 Wh capacity, 6 cells of [5] in series connection, which is able to serve a flight of $30 \mathrm{~km}$. The uppermost graph shows the SOC trace of a randomly selected battery in the battery set. A battery serves multiple delivery requests per day as can be seen from the graph. The second graph in Figure 8 shows the status of each battery of the total battery set we have defined in Section 6. Outside the service hours, all of the batteries are in the available state, while during the service hours, the batteries are in corresponding states. In this case, the number of batteries in available state reaches 0 during the most busy hours and some delivery requests have to wait a bit for a battery to be charged. The third graph in Figure 8 shows the electricity usage from the grid. In this simulation, the batteries are charged with a rate of $2 \mathrm{C}$, and the sum of the charging power reaches up to $900 \mathrm{~kW}$. Inherent losses due to non-ideal battery cycle efficiency and converter losses are also displayed. The last graph
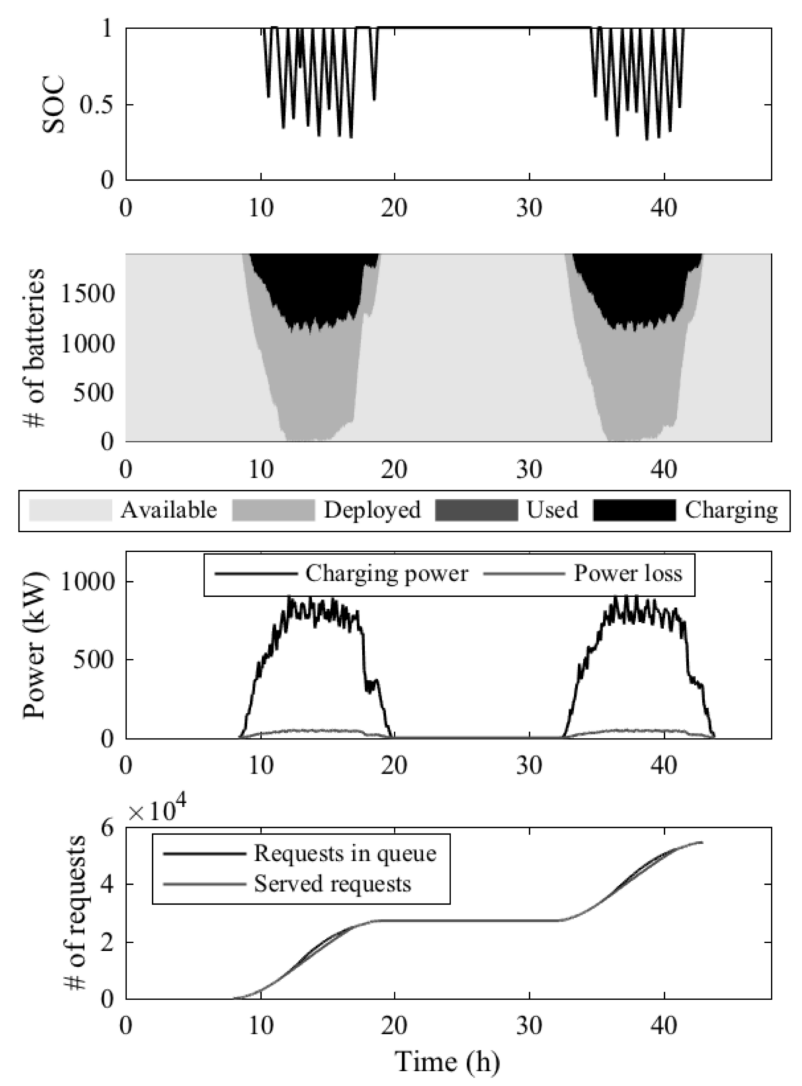

Figure 8: Short-term simulation traces for two days.

shows the number of delivery requests served during the time period. There is a slight, but acceptable lag between delivery request arrivals and actual service time as the number of batteries are not determined for the worst case arrival rate.

Long-term simulation results: The result for a longer time period, two years, is shown in Figure 9. The first graph in the figure shows the SOC swing and capacity degradation of a selected battery. Since the time scale is much larger, not all the details of SOC swing can be seen from the graph, but it is notable that the DoD is generally increasing as the time proceeds and battery ages. In slightly less than a year, the battery reaches its end of life capacity and is no longer used anymore. The second graph shows the packet waiting time, defined in Section 4, changes over the two year period. As the simulation starts with a fresh battery set, no batteries are retired for the first 200 days, and the average capacity is monotonically decreasing. During this time period, there is a clear increasing trend in the average packet waiting time, which coincides with the description given in Section 4. Beyond this time period, battery retirement becomes more like a regular event such that the packet waiting time roughly converges around 15 minutes. The third graph shows the number of retired batteries. The first rush of battery retirement begins around 220 days, and the second rush around 450 days. But as time proceeds, battery retirement rate stabilizes. The last graph shows the final battery capacity distribution. The distribution is almost evenly spread out, with newly replaced batteries around day 700 on the right side of the distribution.

\subsection{Design Space Exploration}

Now, we perform a design space exploration of the infrastructure. The objectives we observe are as follows: The average packet waiting time, initial investment cost, battery replacement cost due to aging, and electricity usage. The control variables for the design 

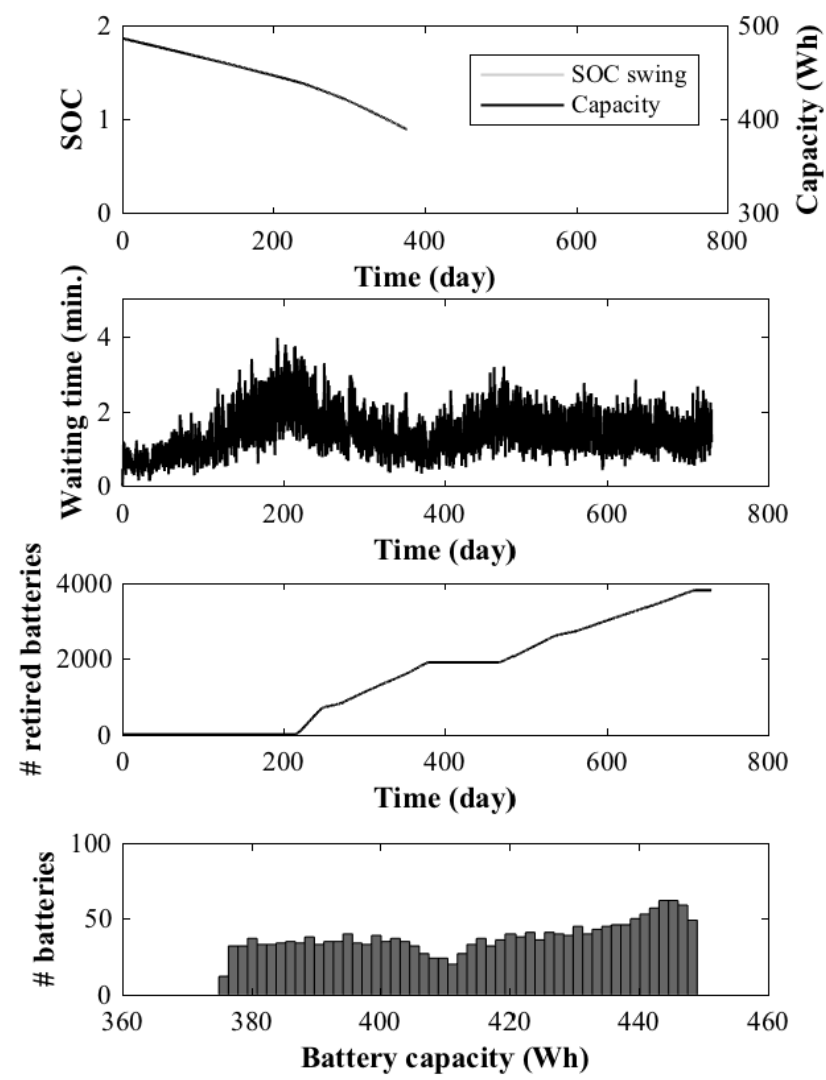

Figure 9: Long-term simulation results over two years.

space exploration are, the number of batteries, use of heterogeneous types of batteries, size of batteries, and battery charging rate. We also take a brief look into the effect of battery attachment policy during runtime.

Impact of number of battery: Figure 10 shows the impact of the total number of batteries and various costs and packet waiting time. We have increased the total number of batteries from 1,700 to 2,200 . The number of batteries below 1,700 resulted in intolerable packet waiting times and was not further investigated. The capacity of the batteries is again 6 cell $452.36 \mathrm{Wh}$ used for the above simulations. We assumed the battery price of $500 \mathrm{USD} / \mathrm{kWh}$ and grid electricity price of 20 cents $/ \mathrm{kWh}$. The exact current market price might be different from the values, but the relative relationship is still valid. Packet waiting time is $451 \mathrm{~s}$ when the number of battery packs is $1,700,87 \mathrm{~s}$ when the number is 1,900 , and rapidly converges to zero beyond this point. Meanwhile, the initial investment cost in batteries and recurring battery replacement cost increases. It is obvious that the initial investment cost increases linearly proportional to the number of batteries. Recurring battery purchasing cost increases from 491k USD to 563k USD. The reason why recurring battery replacement cost increases is the calendar aging of the batteries. As we have stated in Section 4, the calendar aging also plays a significant role and is largely affected by the average SOC of a battery. Thus, it would be a good direction to search for a better runtime algorithms, which makes use of delayed charging similar to a smartphone usage case [15], or charging the battery to just the appropriate amount. The electricity cost remains almost the same, as the actual amount of energy required for the deliveries does not change.

Impact of using heterogeneous sizes of battery: Figure 11 shows the impact of the number of small battery packs on the objectives. We make mixed use of $226.18 \mathrm{Wh}$ batteries together with the

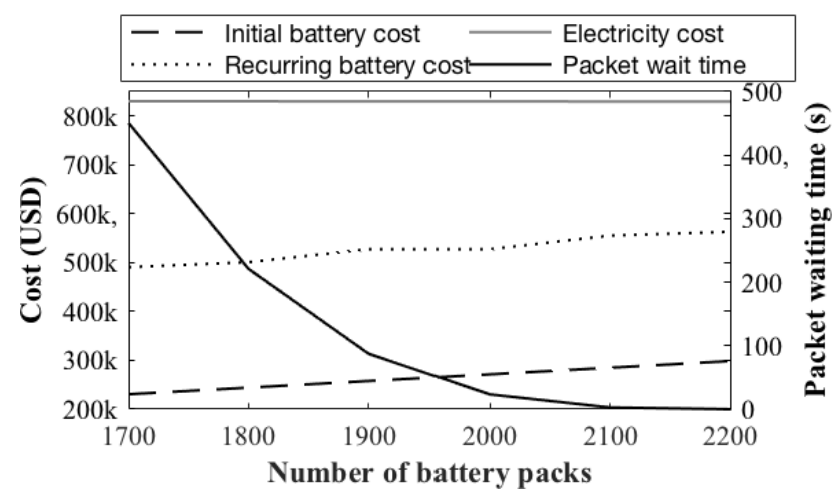

Figure 10: Impact of the total number of batteries on cost and packet waiting time over two years.

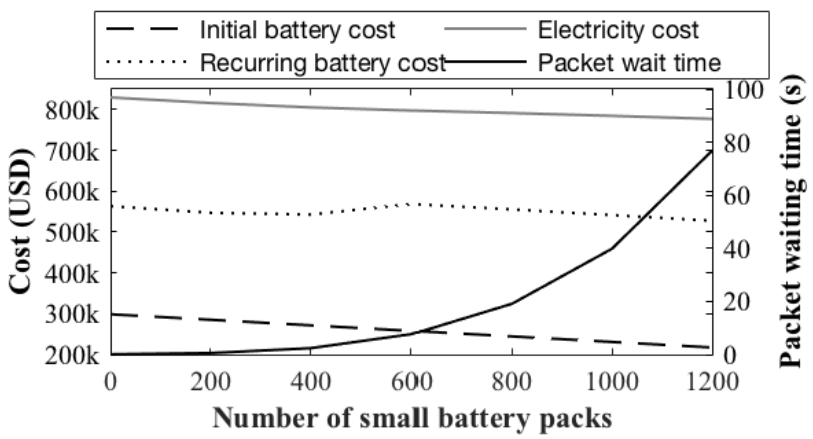

Figure 11: Impact of the portion of small size batteries on cost and packet waiting time over two years.

452.36 Wh battery packs used in the above experiments. Throughout the experiments in Figure 11, the total number of battery packs is maintained to 2,200 . The number of small battery packs corresponds to the value on the $\mathrm{x}$-axis, and the number of larger battery packs is determined to match the total number 2,200. In this experiment, the packet waiting time increases according to the increasing portion of smaller batteries. This is due to the fact that delivery request requiring large amount of energy has to wait for the larger batteries to be available. If the number of larger battery is 0 , some requests will never be served, and hence, the result was excluded from the graph. On the other hand, all the battery related costs decrease according to increased portion of smaller battery packs. It is a trivial fact that the initial battery cost is reduced, as the summed up energy of the whole battery set decreases. The reason the electricity cost reduces is that the drones fly with less battery weight, and thus, it uses less amount of energy per flight. Also, the recurring battery purchase costs decrease as the sum of total battery energy decreases, and drones make more energy efficient flights. The tricky part in evaluating the benefit of using heterogeneous sizes of battery packs is that, it might also be a fair comparison if the total sum of battery capacity is fixed instead of the total number of batteries as in Figure 11. In such case, the result is a combination of Figure 10 and 11 such that packet waiting time is a convex function of the portion of the sum of small battery capacity. Whichever analysis we adhere to, it indicates that having appropriate amount of small battery is beneficial.

Impact of runtime allocation policy: We have implemented two very simple runtime battery attachment policies and made a few simulation runs with heterogeneous battery setup to test whether it has impact on costs. The first policy is random policy, which randomly selects a battery among the ones that satisfy the energy 


\begin{tabular}{cccccc}
\hline \multirow{2}{*}{ Setup } & Policy & $\begin{array}{c}\text { Initial } \\
\text { cost }\end{array}$ & $\begin{array}{c}\text { Elec. } \\
\text { cost }\end{array}$ & $\begin{array}{c}\text { Battery } \\
\text { purchase }\end{array}$ & $\begin{array}{c}\text { Wait } \\
\text { time (s) }\end{array}$ \\
\hline \multirow{2}{*}{ Setup 1 } & Random & 4,750 & 14,595 & 9,718 & 100.32 \\
\cline { 2 - 6 } & Capacity & 4,750 & 14,472 & 10,412 & 96.76 \\
\hline \multirow{2}{*}{ Setup 2 } & Random & 5,428 & 14,456 & 11,106 & 15.49 \\
\cline { 2 - 6 } & Capacity & 5,428 & 14,243 & 11,800 & 12.88 \\
\hline
\end{tabular}

Table 1: Various costs and packet waiting time according to battery attachment policy over two years (cost in USD).

\begin{tabular}{cccccc}
\hline \multirow{2}{*}{ Setup } & $\begin{array}{c}\text { End-of-life } \\
\text { threshold }\end{array}$ & $\begin{array}{c}\text { Initial } \\
\text { cost }\end{array}$ & $\begin{array}{c}\text { Elec. } \\
\text { cost }\end{array}$ & $\begin{array}{c}\text { Battery } \\
\text { purchase }\end{array}$ & $\begin{array}{c}\text { Wait } \\
\text { time (s) }\end{array}$ \\
\hline \multirow{2}{*}{ Setup 1 1} & $80 \%$ & 4,750 & 14,472 & 10,412 & 96.76 \\
\cline { 2 - 6 } & $75 \%$ & 4,750 & 14,439 & 7,678 & 112.56 \\
\hline \multirow{2}{*}{ Setup 2 } & $80 \%$ & 5,428 & 14,243 & 11,800 & 12.88 \\
\cline { 2 - 6 } & $75 \%$ & 5,428 & 14,218 & 8,656 & 15.59 \\
\hline
\end{tabular}

Table 2: Various costs and packet waiting time according to battery end-of-life threshold over two years (cost in USD).

requirement of the delivery task. Drawback of this policy is that unnecessarily large batteries could be assigned to delivery tasks leading to lower energy efficiency. The second policy is capacity policy, which selects the battery with the least surplus energy to serve a task. The results are shown in Table 1. Setup 1 employs 30 large battery packs and 10 small battery packs, while setup 2 employs 30 large battery packs and 20 small battery packs. We have studied a smaller set as capacity policy involved a procedure scanning through all the batteries, therefore causing inefficient simulation. Surprisingly, the impact of battery attachment was not very much significant. Capacity policy was able to reduce the electricity cost as it makes more energy-efficient flights due to reduced battery weight. However, the gain is marginal and it tends to increase the recurring battery purchase costs. We speculate that this is partly due to the side-effect of poorly designed capacity policy that prefers degraded batteries over healthier batteries of same weight as they have less energy margin. Degraded batteries will be exploited even further while healthier batteries tend to be allocated later. Using heterogeneous sizes of batteries already provides significant benefits and the gain of battery attachment strategy is marginal under the current simulation setup. Nevertheless, we do not conclude as the battery attachment policy to be insignificant and leave possibility for improvement with a better designed runtime policy under different battery setups.

Impact of end-of-life capacity threshold: In battery-power vehicle applications, a battery is usually considered to have reached the end-of-life when the capacity becomes $80 \%$ of its initial capacity. The exact threshold value differs from application to application. Throughout the experiments above, we assumed that the threshold is $80 \%$, but setting a different value has significant impact on the objectives. The results are shown in Table 2. Lowering the threshold value to $75 \%$ decreases the recurring battery purchase cost significantly with a marginal increase in the packet wait time. The ratio of recurring battery purchase cost reduction roughly coincides with the ratio of extended lifetime of each battery $(100 \%$ $80 \%=20 \%$ to $25 \%$ ). Assuming that battery aging ratio due to cycle aging is similar for both cases, it is calendar aging that makes difference in the results. Unlike other battery-powered applications such as electric vehicles where the drive-range is significantly affected by the battery degradation, the drone delivery business as a whole suffers less from extended end-of-life.

\section{CONCLUDING REMARKS}

In this paper, we proposed a holistic and detailed analysis on the profitability and time to delivery of the drone delivery busi- ness. While there is increasing attention on the design and navigation techniques of individual drones, this is the first work that addresses the feasibility and profitability of the whole business. We have identified the source of major recurring costs, which are electricity cost and battery purchasing cost, and analyzed how they are affected by design parameters and runtime techniques. Another important parameter to take note is the packet waiting time, which affects time to delivery, a critical metric for the success of such business. The packet waiting time, in general, has a trade-off relationship with the recurring costs, and hence, the design parameters should be carefully chosen. The experimental results on our discrete-event simulator also show that the packet waiting time, and thus, the time to delivery, which is critical to the drone delivery business, is affected by the battery aging. As for the future work, it would be interesting to perform systematic optimization and find the appropriate design parameters and runtime algorithms that balance the operating cost and the time to delivery according to different delivery request patterns.

\section{REFERENCES}

[1] M. Achtelik, J. Stumpf, D. Gurdan, and K.-M. Doth. Design of a flexible high performance quadcopter platform breaking the mav endurance record with laser power beaming. In IEEE/RSJ IROS, 2011.

[2] E. Ackerman. When drone delivery makes sense. IEEE Spectrum, 25, 2014.

[3] Amazon. Amazon prime air. http://www.amazon.com.

[4] P.-J. Bristeau, F. Callou, D. Vissiere, N. Petit, et al. The navigation and control technology inside the ar. drone micro uav. In 18th IFAC world congress, volume 18, pages 1477-1484, 2011.

[5] M. Chen and G. Rincon-Mora. Accurate electrical battery model capable of predicting runtime and I-V performance. IEEE Transactions on Energy Conversion, 21(2), 2006.

[6] R. D'Andrea. Guest editorial can drones deliver? IEEE Transactions on Automation Science and Engineering, 11(3):647-648, July 2014.

[7] J. Engel, J. Sturm, and D. Cremers. Camera-based navigation of a low-cost quadrocopter. In IEEE/RSJ IROS, 2012.

[8] G. M. Hoffmann, H. Huang, S. L. Waslander, and C. J. Tomlin. Quadrotor helicopter flight dynamics and control: Theory and experiment. In Proc. of the AIAA Guidance, Navigation, and Control Conference, volume 2, 2007.

[9] M. Kahlen, K. Valogianni, W. Ketter, and J. van Dalen. A profitable business model for electric vehicle fleet owners. In Smart Grid Technology, Economics and Policies (SG-TEP), 2012 International Conference on, pages 1-5, Dec 2012.

[10] O. Meyer-Rühle. Statistical coverage and economic analysis of the logistics sector in the EU (SEALS). SEALS Consortium, 2008.

[11] Microdrones. MD4-3000 Specifications . http://www.microdrones.com/en/products/md4-3000/technical-data/.

[12] M. O. Milhouse. Framework for autonomous delivery drones. In Proceedings of the 4th Annual ACM Conference on Research in Information Technology, RIIT '15, pages 1-4, New York, NY, USA, 2015. ACM.

[13] A. Millner. Modeling lithium ion battery degradation in electric vehicles. In Proc. CITRES, pages 349-356, 2010.

[14] G. Ning, Z. Zhen, P. Wang, Y. Li, and H. Yin. Economic analysis on value chain of taxi fleet with battery-swapping mode using multiobjective genetic algorithm. Mathematical Problems in Engineering, 2012, 2012.

[15] A. Pröbstl, P. Kindt, E. Regnath, and S. Chakraborty. Smart2: Smart charging for smart phones. In 2015 IEEE 21st International Conference on Embedded and Real-Time Computing Systems and Applications, pages 41-50, Aug 2015.

[16] K. A. Suzuki, P. Kemper Filho, and J. R. Morrison. Automatic battery replacement system for uavs: Analysis and design. Journal of Intelligent \& Robotic Systems, 65(1-4):563-586, 2012.

[17] Technics Consulting. Long range drone dji phantom 3 professional. http://www.technik-consulting.eu/en/optimizing/. 